\title{
DISTRIBUCIÓN GEOGRÁFICA DE LOS NIVELES DE PREVALENCIA DEL CONSUMO DE DROGAS EN POBLACIÓN DE EDUCACIÓN SECUNDARIA EN COSTA RICA, 2015
}

\author{
GEOGRAPHICAL DISTRIBUTION OF PREVALENCE \\ LEVELS DRUG USE IN POPULATION OF HIGH SCHOOL \\ EDUCATION IN COSTA RICA, 2015
}

\author{
William Chacón Serrano ${ }^{1}$ \\ Instituto sobre Alcoholismo y Farmacodependencia, Costa Rica
}

\begin{abstract}
RESUMEN
El consumo de drogas en la población de educación secundaria representa una línea de investigación que ha surgido con más fuerza en los últimos años en Costa Rica, debido al aumento de ingesta de sustancias psicoactivas como el alcohol y la marihuana. Los niveles de prevalencia han experimentado variaciones importantes en los últimos 10 años desde el 2006, se han hecho esfuerzos por realizar un monitoreo del consumo en ésta población; sin embargo, para el estudio del 2015, se incorporaron variables de tipo geográficas que pudieran representar los indicadores de consumo a nivel de provincia y región para dar una perspectiva más amplia del fenómeno en la población costarricense. Se logró evidenciar el comportamiento diferenciado de los niveles de prevalencia en las diferentes provincias, lo que abre el debate de las condiciones por provincia en el comportamiento del fenómeno.
\end{abstract}

Palabras clave: drogas, niveles de prevalencia, adolescentes, sistema de información geográfica.

\section{ABSTRACT}

Drug use in high school population represents a line of research that has emerged more strongly in recent years in Costa Rica due to the increased consumption of drugs like alcohol and marijuana. Prevalence levels have changed considerably in the last 10 years. Since 2006, there have been

1 Licenciado en Ciencias Geográficas, Proceso Investigación. Instituto sobre Alcoholismo y Farmacodependencia (IAFA), San Pedro de Montes de Oca. Costa Rica, Correo electrónico: wchacon@, iafa.go.cr 
William Chacón Serrano. Geographical distribution of prevalence levels drug use in population of high school education in Costa Rica, 2015

efforts to monitor the consumption levels in this population; however, for the 2015 study, geographic variables that could represent the consumption indicators at province and region level were included to give a broader perspective of the phenomenon in the Costa Rican population. It was possible to show the differentiated behavior of prevalence levels of consumption in different provinces, thus opening the debate on the conditions by province regarding the phenomenon behavior.

Keywords: drugs, prevalence levels, adolescents, geographic information system

\section{Introducción}

El presente artículo se enfoca en el análisis de comportamiento del consumo de drogas en la población de educación secundaria de Costa Rica para el año 2015, mediante el indicador de prevalencia del consumo de drogas, segmentado por provincia y regiones del país. Estos resultados son el producto de la cuarta investigación que se realiza desde el 2006, contribuyendo a la continuidad de estudios en población escolar elaborados por el Instituto sobre Alcoholismo y Farmacodependencia.

La ingesta de drogas es un fenómeno social que se presenta como parte de las dinámicas de convivencia social y afecta a la población en la medida que éste consumo avanza a un patrón abusivo, pasando a la enfermedad de la dependencia, la cual se asocia a las necesidades de consumir drogas, generando diferentes problemáticas individuales y grupales.

Los adolescentes son uno de los grupos afectados por el uso y abuso de drogas, especialmente por el alcohol, tabaco y marihuana, aspectos que se han puesto en evidencia en estudios recientes realizados por la Oficina Contra las Drogas y el Delito (UNODC, 2016, p.11).

Ante factores como la alta disponibilidad, las relaciones interpersonales, la baja percepción de riesgo al uso de sustancias, cambios emocionales e incluso dinámicas familiares, se produce una combinación de factores que potencializan el contacto con las sustancias, donde el factor que preocupa es la permanencia en el uso de las diferentes drogas.

Los niveles de prevalencia del uso y abuso de drogas en etapa temprana es alta, por lo cual se tiene la consigna que los adolescentes tiene que aprender a convivir con las drogas, donde en muchos casos se debe decidir entre abstinencia y consumo a lo cual las decisiones juegan un papel importante. Asimismo, comprender estas dinámicas sociales representa un paso importante para el abordaje y fortalecimiento de las intervenciones preventivas en la mejora de esas relaciones entre adolescentes y las llamadas drogas de abuso. 
Como parte del trabajo de indagación que se lleva a cabo en el proceso de investigación del Instituto sobre Alcoholismo y Farmacodependencia (IAFA), se plantea la necesidad de analizar los indicadores de prevalencia de consumo de drogas por medio de la asociación con espacios geográficos en Costa Rica. Para llevar a cabo este análisis se echó mano de los Sistemas de información Geografica (SIG) como herramienta tecnológica que pudiera aportar en la mejora de la presentación de información, además, que contribuyera en la interacción con variables de salud para el estudio del fenómeno social (Ramirez, 2009). Los resultados presentados se enmarcan en las provincias del país y las regiones administrativas del IAFA, las cuales presentan características diferentes de consumo de drogas que vale la pena destacar en el presente artículo.

Los niveles de prevalencia muestran un comportamiento diferenciado por provincia y región en Costa Rica que evidencian las principales drogas consumidas por la población estudiantil de secundaria. Asimismo, se destaca dentro de los hallazgos más importantes la disminución de la percepción de riesgo del consumo de marihuana y alcohol en los jóvenes estudiantes que han dado como conclusión el aumento en el uso de estas dos sustancias principalmente.

\section{Material y Métodos}

La muestra de esta investigación se seleccionó a partir de un listado (marco muestral) de los colegios públicos y privados existente en el Ministerio de Educación Pública para el año 2014. Fueron seleccionados en forma aleatoria cincuenta centros educativos y en cada uno de ellos una sección por nivel educativo para un total de 263 secciones. En cada sección se entrevistó al total de estudiantes presentes el día y hora en que se efectuó la recopilación de los datos. La muestra efectiva estuvo conformada 5.863 informantes (véase la Tabla 1).

La lista de matrícula para el año 2014 contenía 958 centros educativos de educación secundaria en modalidad regular. La matrícula total en esos centros educativos fue de 369.573 estudiantes (183.196 hombres y 186.377 mujeres).

La siguiente tabla muestra el número de centros educativos participantes por provincia. 
Tabla 1. Ubicación y cantidad de estudiantes entrevistadosen los centros educativos seleccionados, Costa Rica, 2015.

\begin{tabular}{|l|c|c|}
\hline \multicolumn{1}{|c|}{ Provincia } & Número de colegios & Muestra de estudiantes \\
\hline San José & 12 & 1484 \\
\hline Alajuela & 10 & 1178 \\
\hline Cartago & 5 & 739 \\
\hline Heredia & 5 & 542 \\
\hline Guanacaste & 5 & 583 \\
\hline Puntarenas & 8 & 793 \\
\hline Limón & 5 & 544 \\
\hline Total & $\mathbf{5 0}$ & $\mathbf{5 8 6 3}$ \\
\hline
\end{tabular}

Fuente: IAFA, 2015.

\subsection{Instrumento}

El cuestionario utilizado consta de 109 preguntas, distribuidas en seis secciones, que se mencionan a continuación:

a) Información personal y académica,

b) Percepción del riesgo por consumo de drogas,

c) Consumo de drogas por parte del estudiante,

d) Exposición a la oferta y curiosidad por el consumo de drogas,

e) Consumo familiar y percepción de seguridad,

f) Involucramiento parental,

g) Aspectos relacionados sobre conducción, relaciones sexuales y consumo de drogas.

Específicamente para esta investigación se tomó en cuenta únicamente los aspectos $\mathrm{B}$ y $\mathrm{C}$ relacionados con el consumo de drogas, analizados desde el análisis provincial y regiones administrativas del IAFA en Costa Rica.

La recolección de los datos se llevó a cabo durante el segundo semestre lectivo del año 2015. El desglose por provincia y nivel académico de los estudiantes seleccionados en la muestra de 5.863 aparece en la siguiente Tabla: 
Tabla 2.Estudiantes entrevistados por nivel académico y provincia Costa Rica, 2015

\begin{tabular}{|c|c|c|c|c|c|c|c|c|}
\hline \multirow{2}{*}{ Provincia } & \multirow{2}{*}{$\begin{array}{l}\text { Total de } \\
\text { colegios }\end{array}$} & \multicolumn{6}{|c|}{ Estudiantes por nivel } & \multirow{2}{*}{$\begin{array}{c}\text { Total de } \\
\text { estudiantes }\end{array}$} \\
\hline & & $7^{\circ}$ & $8^{\circ}$ & $9^{\circ}$ & $10^{\circ}$ & $11^{\circ}$ & $12^{\circ}$ & \\
\hline San José & 12 & 274 & 285 & 275 & 253 & 311 & 86 & 1484 \\
\hline Alajuela & 10 & 229 & 203 & 240 & 237 & 198 & 71 & 1178 \\
\hline Cartago & 5 & 135 & 145 & 136 & 131 & 131 & 61 & 739 \\
\hline Heredia & 5 & 93 & 91 & 91 & 104 & 114 & 49 & 542 \\
\hline Guanacaste & 5 & 113 & 123 & 117 & 104 & 96 & 30 & 583 \\
\hline Puntarenas & 8 & 144 & 145 & 138 & 143 & 143 & 80 & 793 \\
\hline Limón & 5 & 82 & 96 & 109 & 101 & 108 & 48 & 544 \\
\hline Total & 50 & 1070 & 1088 & 1106 & 1073 & 1101 & 425 & 5863 \\
\hline
\end{tabular}

Fuente: IAFA, 2015.

Durante la aplicación de los cuestionarios no se presentaron rechazos y no fue necesario descartar alguno por la presencia de inconsistencias reiteradas.

\section{Indicadores más importantes}

Son indicadores estándar a nivel internacional que permiten realizar comparaciones de los patrones de consumo de forma simultánea y cronológica, los mismos dan cuenta del porcentaje de consumidores de cada sustancia o de conjuntos relevantes de ellas.

Los porcentajes de consumidores hay tres períodos de referencia que son de particular interés a nivel internacional y de los que se informa en este documento. El primero de ellos alude al consumo alguna vez en la vida. Su importancia radica en el hecho de que el riesgo de sufrir trastornos o daños por la ingesta de una droga se reduce a cero en una persona que nunca ha ingerido esa droga.

El segundo período para calcular los porcentajes de consumidores de los doce meses anteriores al momento en que se responde al cuestionario, es decir al consumo del último año. Permite discriminar el inicio en el consumo de una droga de un uso reciente y pone en evidencia a los consumidores de la misma que luego de haberla probado perseveran en el consumo.

Finalmente, el último período de referencia es el del mes anterior al día en que se hace la entrevista. Si bien puede estar influido por hechos que 
ocurran en la zona donde residan algunos estudiantes coincidiendo con el tiempo que tarde la recolección de los datos, se considera un indicador relevante para aproximar al total de estudiantes que muestran un uso regular o frecuente de alguna droga.

Los análisis se efectuaron valiéndose de las aplicaciones PASW Statistics versión 23, STATA y Excel de Microsoft, así como el ArcGis 10.2.

\section{Consumo de drogas en Población escolarizada}

El consumo de drogas en la población adolescente son uno de los aspectos donde los estudios científicos han puesto más énfasis a la hora investigar la relación entre las drogas y la juventud. En este periodo de cambios físicos, psicológicos y madurez emocional que enfrentan los adolescentes, se presentan una serie de retos asociados al consumo de drogas, y que son determinados por las mismas condiciones de desarrollo de las personas (Gonzales et al, 1996, p.5). Empezando por su propia concepción, las Naciones Unidas han definido la adolescencia como el periodo que va entre los 10 a 19 años (UNICEF, 2011, p 8), donde se enfrenta a una serie de cambios y adaptaciones diversas que han evidenciado lo difícil de enmarcar una definición para "adolescente" que incluso en la actualidad es difícil de definir. La legislación de los países, los aspectos culturales, los derechos humanos de las personas, entre muchos otros factores, han puesto de relieve la complejidad de definir adecuadamente el término. Sin embargo, se deja claro que a pesar de estas complejidades, todas estas personas menores de edad tienen derechos inscritos en las convenciones de derechos humanos que los hace una población respaldada internacionalmente y por la legislación de cada país (UNICEF, 2011, p. 20).

La población adolescente representa un inicio en el contacto con las diferentes sustancias tanto licitas como ilícitas que existen en una sociedad, donde influyen muchos factores que potencializan el uso de las sustancias. Según Salamó et al (2010, p.5) la población experimenta el primer consumo de drogas en la etapa de adolescente, ya sea por factores individuales o sociales. La edad inicio muestra que ese primer contacto con una determinada sustancia se da en edades muy tempranas, acelerando los problemas que esta acción podría traer a su desarrollo futuro. Para reforzar esta afirmación, los informes sobre consumo de drogas en países de America Latina han puesto de relieve que las personas menores de edad 
se inician en el contacto con las drogas en edades de 12 años en adelante; es decir, en edades donde se encuentran en el sistema educativo (CICAD/ OEA, 2015, p. 8). En países como Argentina el porcentaje de adolescentes entre los 12 y 17 años que han consumido marihuana en el último año y último mes fue de $8,4 \%$ y $4,2 \%$ respectivamente (OAD, 2014).

En esta misma línea, otros estudios internacionales han demostrado que la población escolarizada se enfrenta a factores de riesgo del consumo de sustancias psicoactivas, (Nazar et al, 1994, p. 4); es decir, la escolaridad, uso en la familia, amigos consumidores, entre otros aspectos han sido señalados como factores de riesgo en el uso de los adolescentes. Asimismo, también se han estudiado y demostrado que existen factores de protección para los adolescentes, tal es el caso de encontrase en el sistema educativo, recibir involucramiento de los padres, actividades culturales, entre otras, donde se potencializan oportunidades de desarrollo para los jóvenes, lo que ha atrasado la edad de inicio en el consumo (Gonzales et al, 1996, p. 7).

\section{Factores asociados al consumo}

Los factores de riesgo que incentivan el consumo de drogas en los adolescentes han sido desarrollados de diversas maneras, por un lado los contextos en los que se desenvuelven los jóvenes, ya sea recreativos, educativos y familiares, entre otros han generado importantes hallazgos en la problemática. Según autores como Nazar et al. (1994) en la familia, los aspectos individuales, la edad, buscar nuevas sensaciones e identidad, así como la presión de pares, han sido mencionado como factores de riesgo en el consumo de sustancias psicoactivas (p.6). Se ha comprobado que uno de los principales aspectos negativos que tienen mayor probabilidad de consumo en la población juvenil tiene relación con tener amistades que consuman alguna droga o drogas; además, los factores familiares han jugado un papel importante en este tipo de fenómenos sociales, el consumo en algún familiar específicamente en el padre, según este mismo estudio antes mencionado, no fue tan representativo como el de poseer amigos consumidores, aunque, sí representó de los más sobresalientes en la influencia del uso de drogas (Salamó et al, 2010).

Por otra parte, el caso de poblaciones adolescentes en problemas judiciales para el caso de Costa Rica, el contacto con las sustancias 
psicoactivas se presentó de forma exponencial, según Fonseca y Bejarano (2012) la población menor de edad privada de libertad reveló porcentajes más elevados en las principales drogas ilícitas. El contacto con drogas como la Cocaína y el Crack se presentaron para el año 2008 en un 37\% y un $34 \%$ respectivamente, estos porcentajes se refieren a los que indicaron haber consumido "alguna vez" en la vida. Por el contrario en adolescentes que se encontraban en el sistema educativo en el año 2009 el porcentaje de contacto con las sustancias fue de $0,025 \%$ Cocaína y $0,0125 \%$ Crack (Bejarano et al, 2011, p. 80). En ese sentido vale la pena mencionar que el tratamiento y la demanda especializada para personas menores de edad con problemas por uso de sustancias presentó un ligero aumento al pasar de 354 personas registradas en el 2011 a 450 para el 2012 (IAFA, 2014)

Por último, y no menos importante cabe señalar que la percepción de riesgo es otro de los elementos más importantes estudiados en la actualidad, ya que se ha indicado en estudios pasados en países como España (Salamó et al, 2010, p. 8). Una baja percepción de riesgo, el consumo de sustancias como el alcohol, donde se podría asociar la ingesta de grandes cantidades de este con esta disminución de peligrosidad. Según Fonseca et al, (2012) para el caso de Costa Rica, los estudiantes de secundaria también han mostrado una baja percepción de riesgo al consumo de drogas como el alcohol, tabaco marihuana (p. 40), lo que deja en evidencia que este factor es muy perceptivo para el análisis del consumo de drogas en los jóvenes y que se ha pasado de tener sustancias con una percepción de riesgo alto a menor riesgo, según lo demuestran los estudios.

\section{Resultados}

El análisis de los niveles de prevalencia del consumo de drogas, se presentó mediante la distribución geográfica provincial, y regiones administrativas del IAFA en el país, donde también se tomó en cuenta la segmentación por sexo del indicador en los estudiantes entrevistados, de esta forma se permitió visualizar las tendencias del consumo de las tres principales drogas consumidas por los estudiantes de secundaria en Costa Rica (alcohol, tabaco y marihuana). 


\section{Niveles de prevalencia por Provincias}

A partir de los resultados obtenidos se realizaron los mapas de síntesis de la información de los indicadores antes mencionados (vida, año y mes), en la figura 1 se presenta la información del indicador de alguna vez en la vida de las tres drogas principales distribuidas por provincias en Costa Rica.

Figura 1. Consumo de alguna vez en la vida de alcohol, tabaco y marihuana según población de educación secundaria por provincia en Costa Rica, 2015.

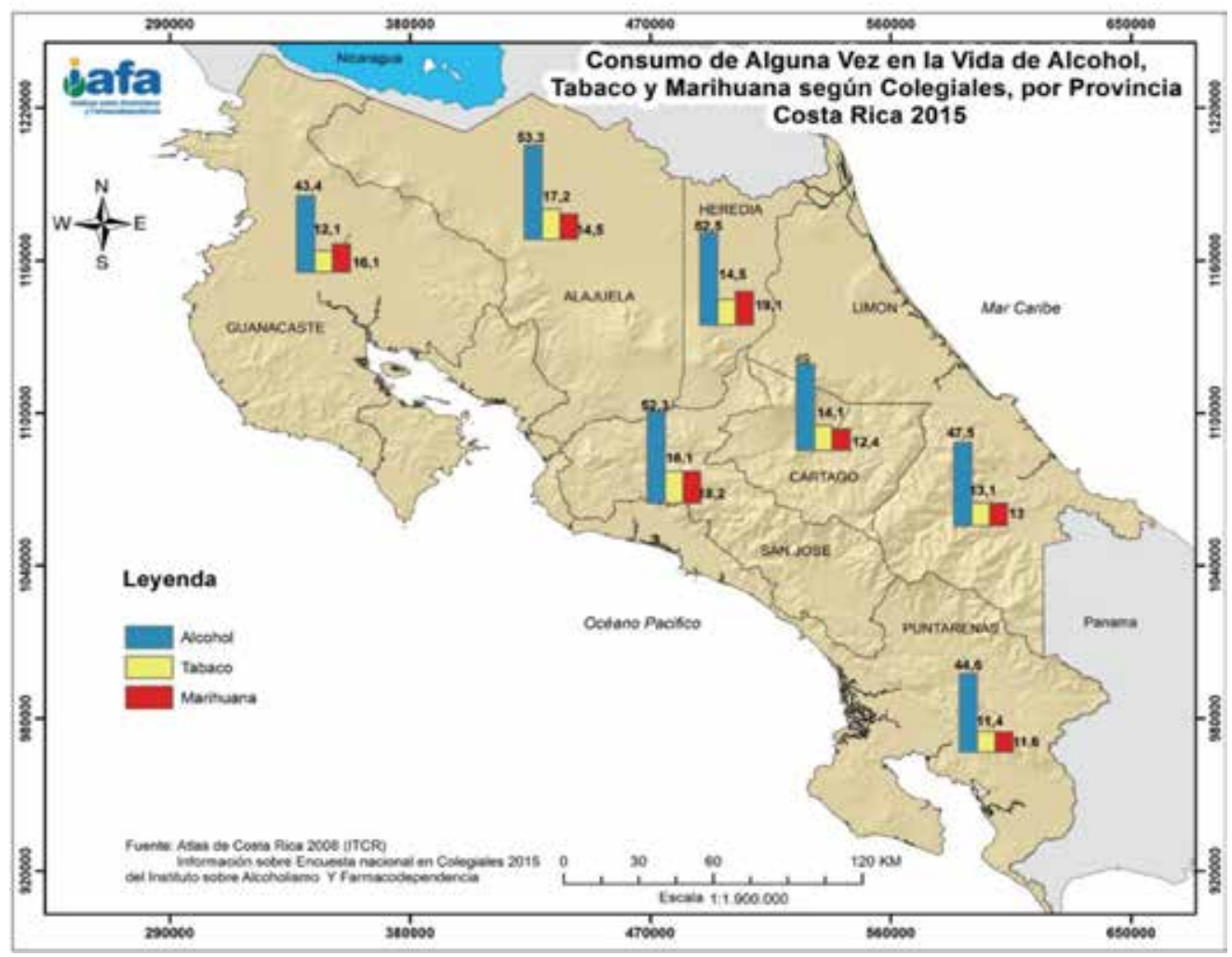

Fuente: IAFA, 2015.

Según se aprecia, el alcohol es la droga que más han consumido alguna vez en la vida los estudiantes de secundaria, en todas las provincias los porcentajes rondan entre un $43 \%$ a $53 \%$ de personas que afirmaron 
haber probado esta sustancias psicoactiva por lo menos alguna vez. Seguido del alcohol se ubica en segundo lugar el tabaco en cada provincia a excepción de Heredia donde se logró comprobar que la marihuana se encuentra como segunda droga de experimentación para el estudiantado. En cuanto al consumo de alguna vez en la vida en cada una de las provincias en comparación con el estudio realizado por parte del IAFA (2014) se pudo determinar que en el caso de la marihuana esta aumentó en todas las provincias. En el caso de San José para el 2012 la prevalencia era de $16,4 \%$ mientras que para el 2015 se estableció en $18,1 \%$ tal y como lo muestra el mapa anterior, en el caso de Heredia los indicadores estimados fueron $17,5 \%$ vrs $19,1 \%$ para los años 2012 y 2015 respectivamente. Las provincias costeras pasaron de $12,4 \%$ y 9,9\% Limón y Puntarenas respectivamente en el año 2012 a $13 \%$ y $11,6 \%$ en el 2015 .

Para el caso de Guanacaste esta provincia registró un aumento importante en el consumo de marihuana pasando de 9,4\% en el 2012 a $16 \%$ en 2015. Por último, la provincia de Cartago reportó tener una prevalencia de 15,8\% en el 2012, la cual pasó a 12,4\% en el 2015.

Con respecto al consumo del último año para las drogas seleccionadas, se presenta el siguiente mapa (figura 2), donde se resume el comportamiento del indicador por provincia. Cabe destacar que en este indicador la diferencia entre el mayor consumo de marihuana con respecto al tabaco se hace más notoria 
Figura 2. Consumo del último año de alcohol, tabaco y marihuana según población de educación secundaria por provincia en Costa Rica, 2015.

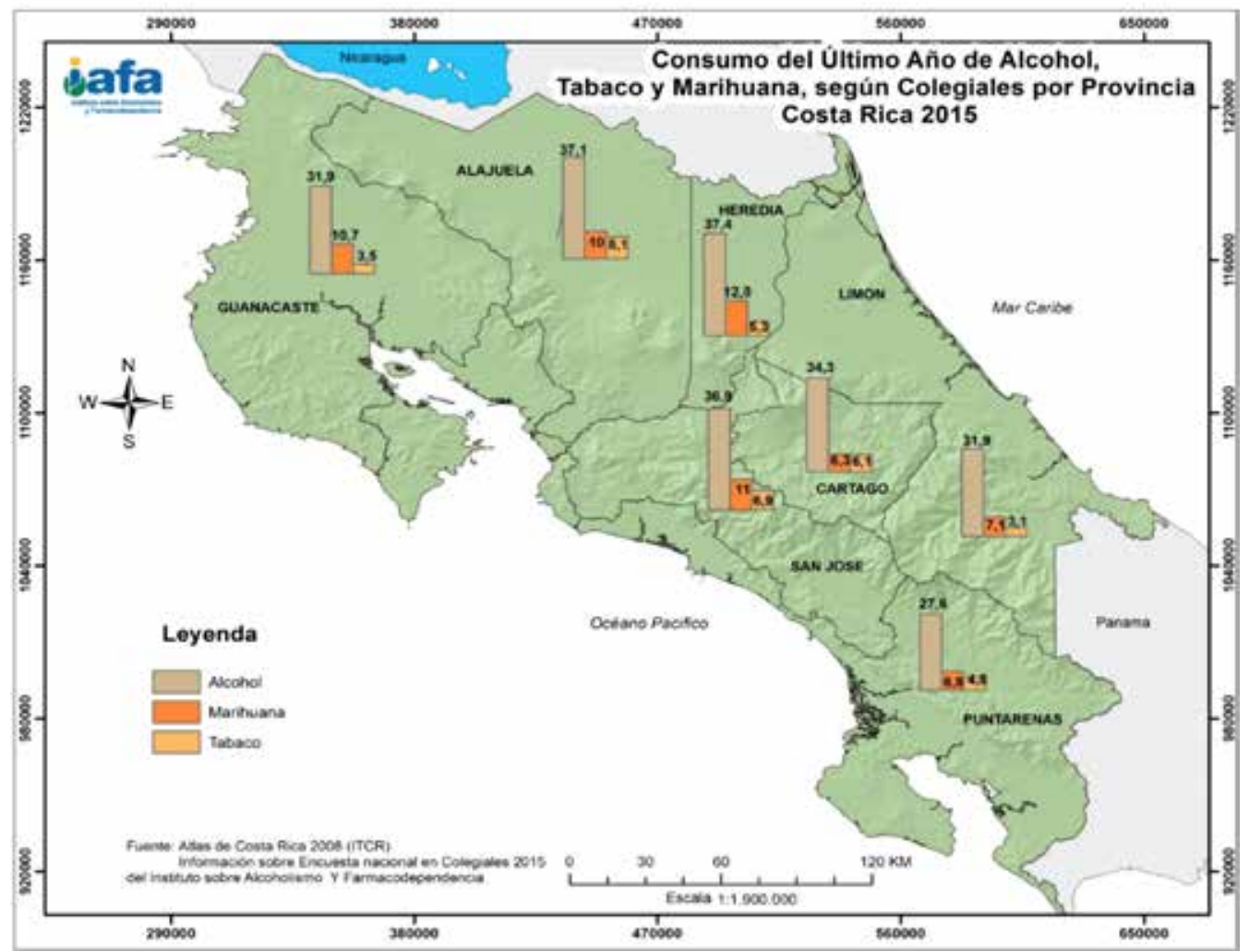

Fuente: IAFA, 2015.

$\mathrm{Al}$ analizar la tendencia del indicador de acuerdo con cada provincia, se aprecia que el último año refleja una propensión al alcohol como droga principal de consumo por parte de los adolescentes, donde además, pasó a segundo lugar en importancia el consumo de marihuana y no el tabaco tal y como se había mantenido en estudios anteriores. Los adolescentes reportaron que el tabaco ha sido menos consumido, fenómeno que hasta el 2015 no se evidenciaba. Por el contrario, el tabaco había sido junto con el alcohol la droga de preferencia.

Según se había mencionado en el año 2012, el estudio de colegiales, recopiló información donde todavía el tabaco se presentaba como la segunda droga de prioridad para el consumo en los adolescentes (IAFA, 
William Chacón Serrano. Geographical distribution of prevalence levels drug use in population of high school education in Costa Rica, 2015

2014); sin embargo, para el 2015 la marihuana lo desplazó en todas las provincias, lo cual se ve más reflejado en el uso del último mes o también conocido como consumo activo.

En la figura siguiente se detalla este comportamiento del consumo de drogas del último mes en la población juvenil.

Figura 3. Consumo del último mes de alcohol, tabaco y marihuana según población de educación secundaria por provincia en Costa Rica, 2015.

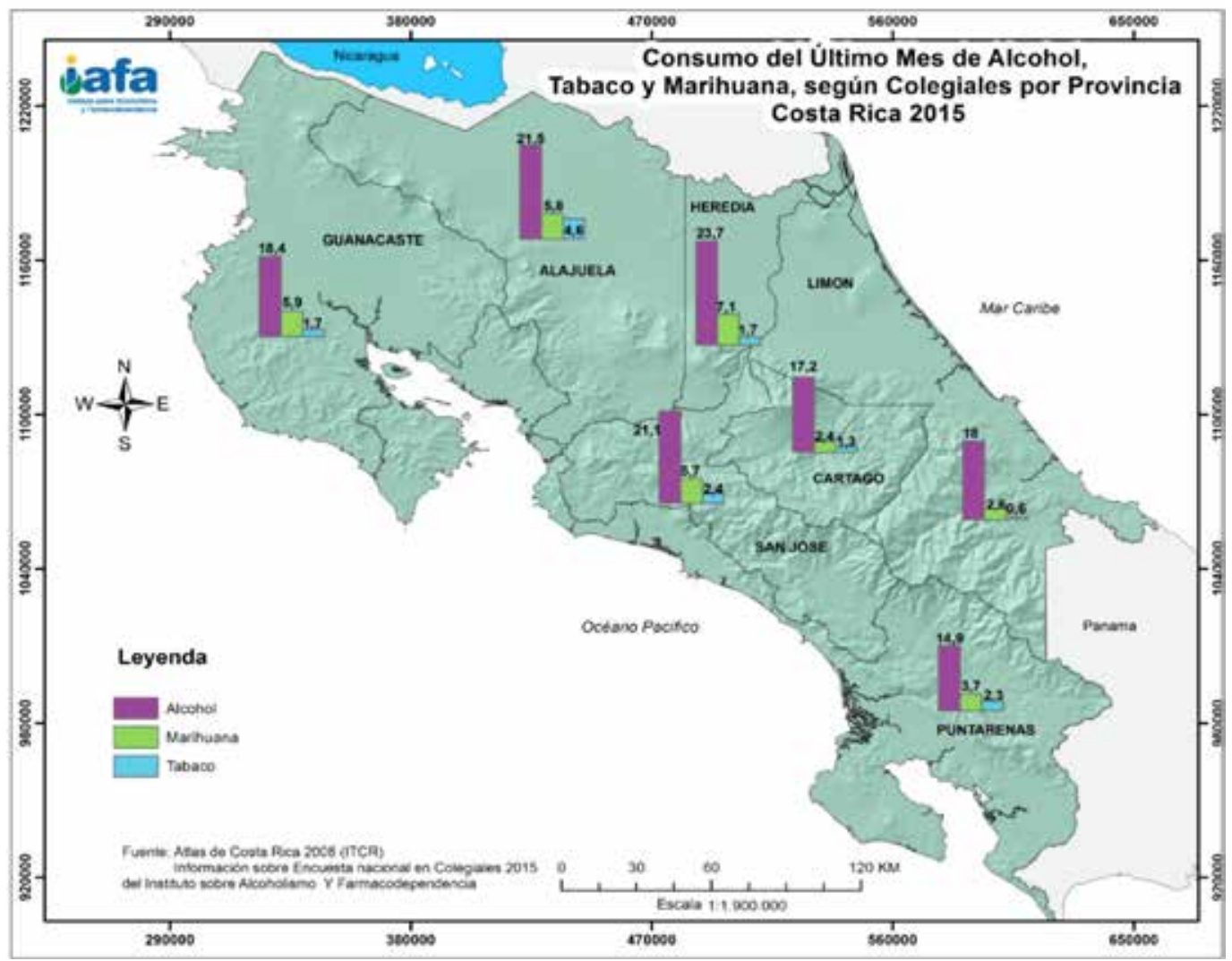

Fuente: IAFA, 2015.

El mapa anterior permite apreciar la ingesta en los últimos 30 días, o también conocido como consumo activo. Los porcentajes en prácticamente todas las provincias superan el $15 \%$ de personas menores de edad que ingieren activamente el alcohol y que este comportamiento en muchos 
casos está asociado a un uso riesgoso; es decir, consumo en los cuales se exponen a lesiones físicas, violencia, y hasta lesiones psicológicas entre otros factores negativos.

Siguiendo en esta línea, sobre el patrón riesgoso de ingesta de alcohol supone un patrón de ingesta etílica que aumenta el riesgo de que se produzcan consecuencias negativas tanto para el bebedor como para las personas que los rodean (Babor, Higgins-Biddle, Saunders y Monteiro, 2001) en este caso en los adolescentes se presenta un riesgo mayor al exponerse a cantidades importantes de alcohol en contextos donde su salud se vería seriamente afectada.

Asimismo, según los resultados, la marihuana y tabaco a pesar de mostrar valores no superiores al $10 \%$, son indicadores claros del aumento en el consumo en algunas provincias como Heredia y Alajuela, ya que el consumo activo de marihuana se sitúo en $7,1 \%$ y 5,8 respectivamente, valores por encima de provincias como Limón $(2,6 \%)$ o Puntarenas $(3,7 \%)$.

\section{Niveles de prevalencia por regiones del IAFA}

El mapa siguiente muestra la división territorial en las cuales se distribuyó la muestra del estudio de colegiales para el trabajo de recolección de datos. En esta segunda parte se detalla la información obtenida por las regiones administrativas del IAFA. La segmentación cuenta con 10 regiones, las cuales se detallan en relación con los resultados obtenidos. 
William Chacón Serrano. Geographical distribution of prevalence levels drug use in population of high school education in Costa Rica, 2015

Figura 4. División regional del Instituto sobre Alcoholismo y Farmacodependencia Costa Rica, 2015

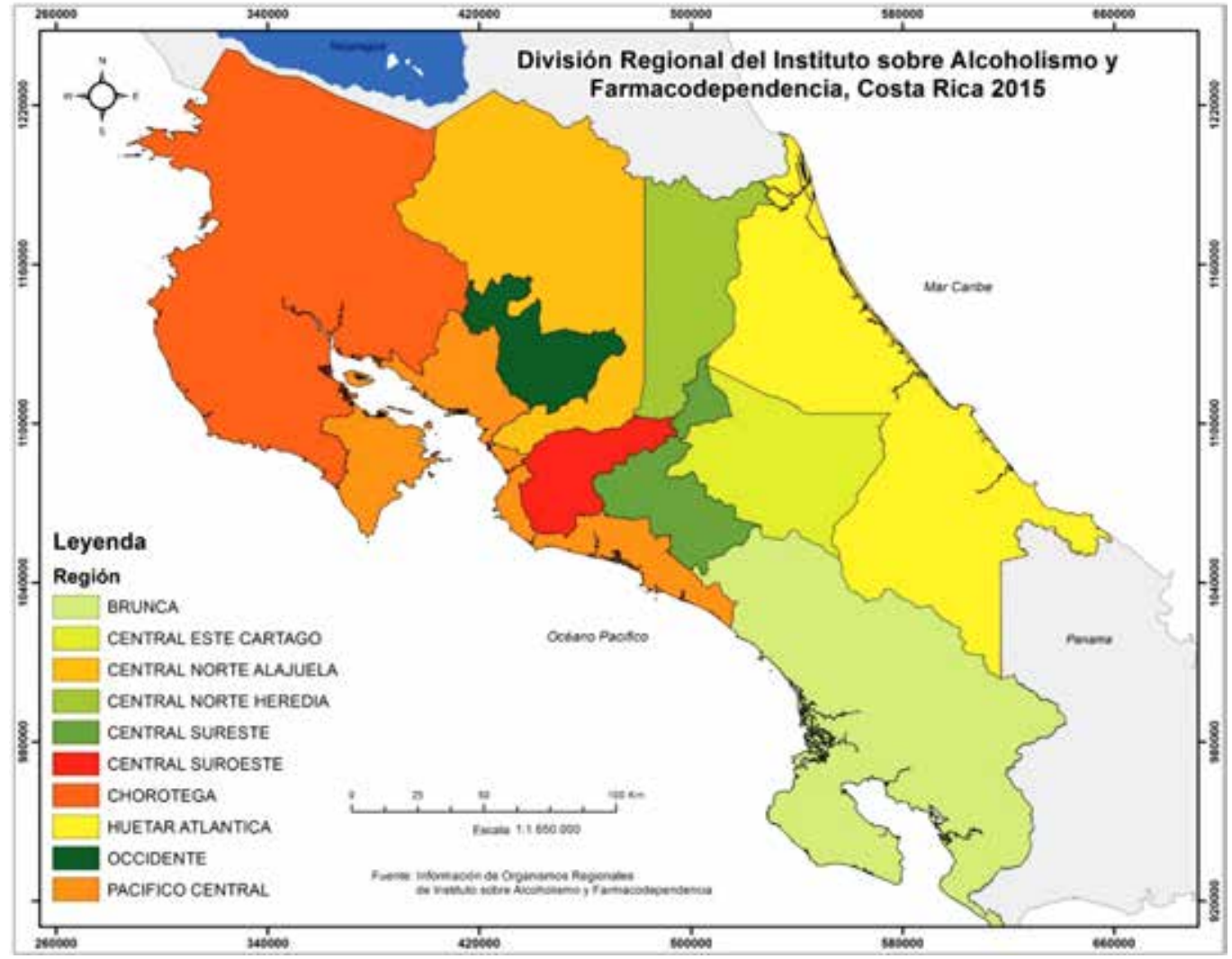

Fuente: IAFA, 2015.

En este apartado se analiza la información de los indicadores por cada droga estudiada y se resumen los porcentajes de prevalencia del consumo de drogas por regiones y se comparan.

El gráfico siguiente muestra la información de los niveles de prevalencia del tabaco en cada región de estudio. En él se pueden analizar aspectos importantes y al mismo tiempo potenciar nuevos temas de investigación en la temática de regiones. 
Gráfico 1. Niveles de prevalencia del consumo de tabaco en colegiales, según regiones del IAFA en Costa Rica, 2015

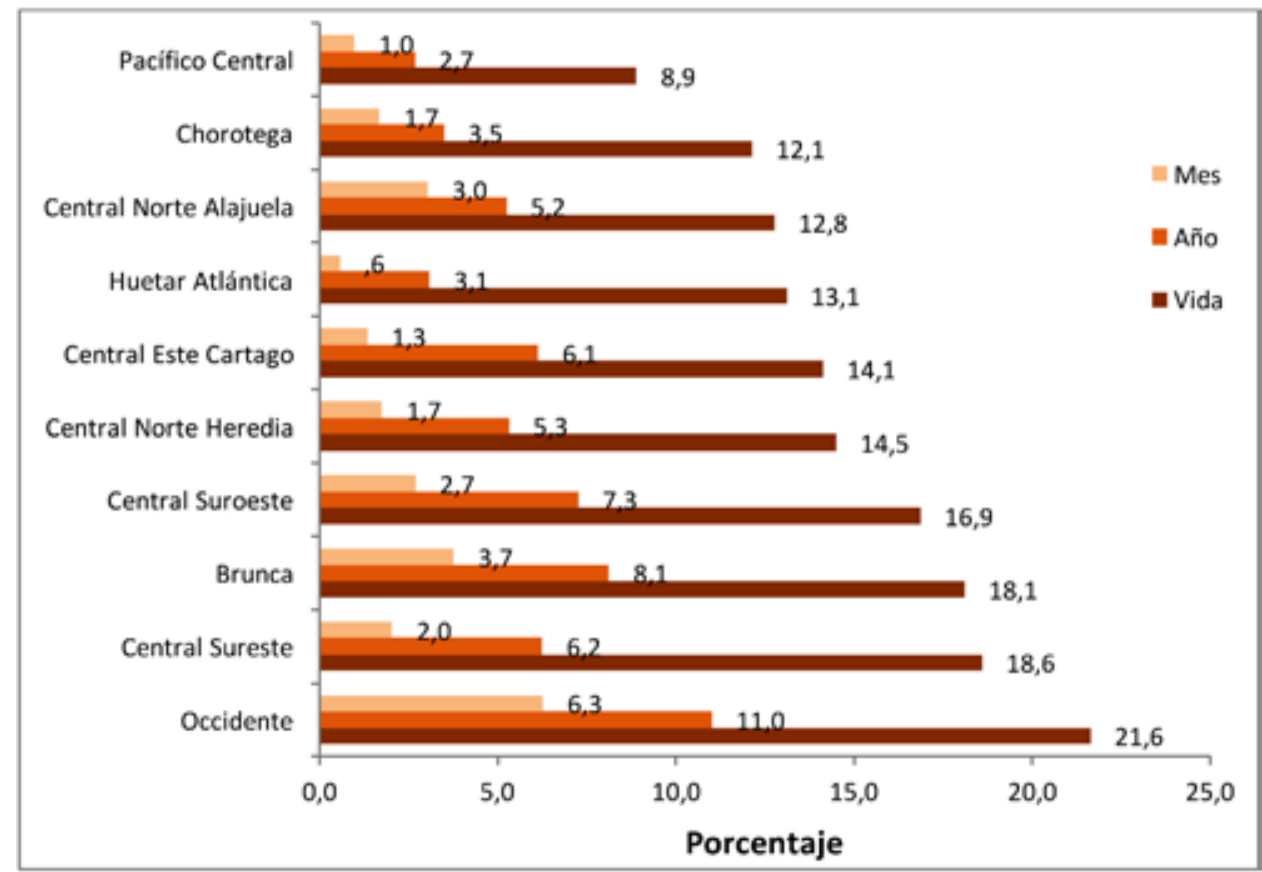

Fuente: IAFA, 2015.

Los datos de la figura anterior, evidencia que existe una diferencia en el consumo de tabaco por regiones que llama la atención de destacar. Por un lado, se denotan porcentajes de alguna vez en la vida que duplican de una región a otra, caso de la región Occidente $(21,6 \%)$, la cual contrasta con la Pacifico Central $(8,9 \%)$, y por otro lado se aprecian regiones donde los valores son muy parecidos en este caso la región Central Sureste y la Brunca con porcentajes de $18,6 \%$ y 18,1 respectivamente.

En cuanto al análisis por región, es importante mencionar que para el 2011 según el Índice de Desarrollo Humano Cantonal (IDHc) (PNUD/ UCR, 2011) los cantones de la región occidente; es decir, San Ramón, Grecia, Palmares presentaron unos índices ubicados en las categorías medio alto, lo que genera cuestionamientos en cuanto a la relación entre el consumo de drogas y las características sociales de las regiones o del territorio en estudio. 
En el caso particular del alcohol, los resultados de la encuesta mostraron estimaciones que reflejan el mayor consumo de esta sustancia en los adolescentes, donde los porcentajes se mantienen entre el $43,4 \%$ y el $55 \%$ sobre consumo de alguna vez en la vida, el siguiente gráfico muestra detalladamente la información para cada región.

Gráfico 2. Niveles de prevalencia del consumo de alcohol en colegiales, según regiones del IAFA en Costa Rica, 2015

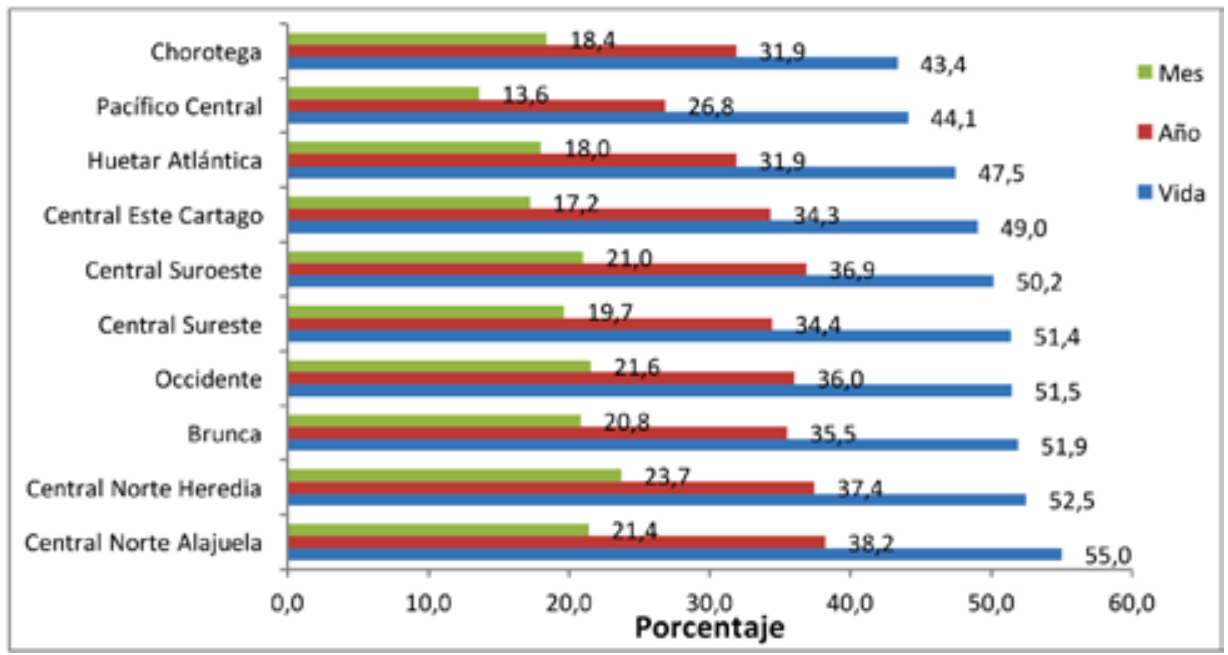

Fuente: IAFA, 2015.

Según se aprecia en la información anterior, las regiones ubicadas al norte del país (Alajuela y Heredia) presentan los mayores porcentajes de consumo de alcohol tanto en el consumo en la vida como el consumo del último año, igualmente seguido de la región occidente.

En cuanto a consumo del último mes, Heredia se mantiene como la región con el indicador más alto $(23,7 \%)$, un porcentaje bastante elevado si se tiene en cuenta que muchos de los estudiantes que consumen alcohol en esas edades tienden a tener una ingesta de riesgo. Los resultados revelan que al menos un grupo importante de personas (20,2\% mujeres y $21,4 \%$ hombres) mencionaron haber andado en un automóvil por lo menos alguna vez en el último año con una persona que había ingerido bebidas alcohólicas. Llama la atención este grupo de estudiantes que han mantenido conductas de riesgo relacionadas con el alcohol. 
Por último, se analizó el uso de marihuana en la población estudiantil por región, donde se describen a continuación los resultados.

Gráfico 3. Niveles de prevalencia del consumo de marihuana en colegiales, según regiones del IAFA en Costa Rica, 2015

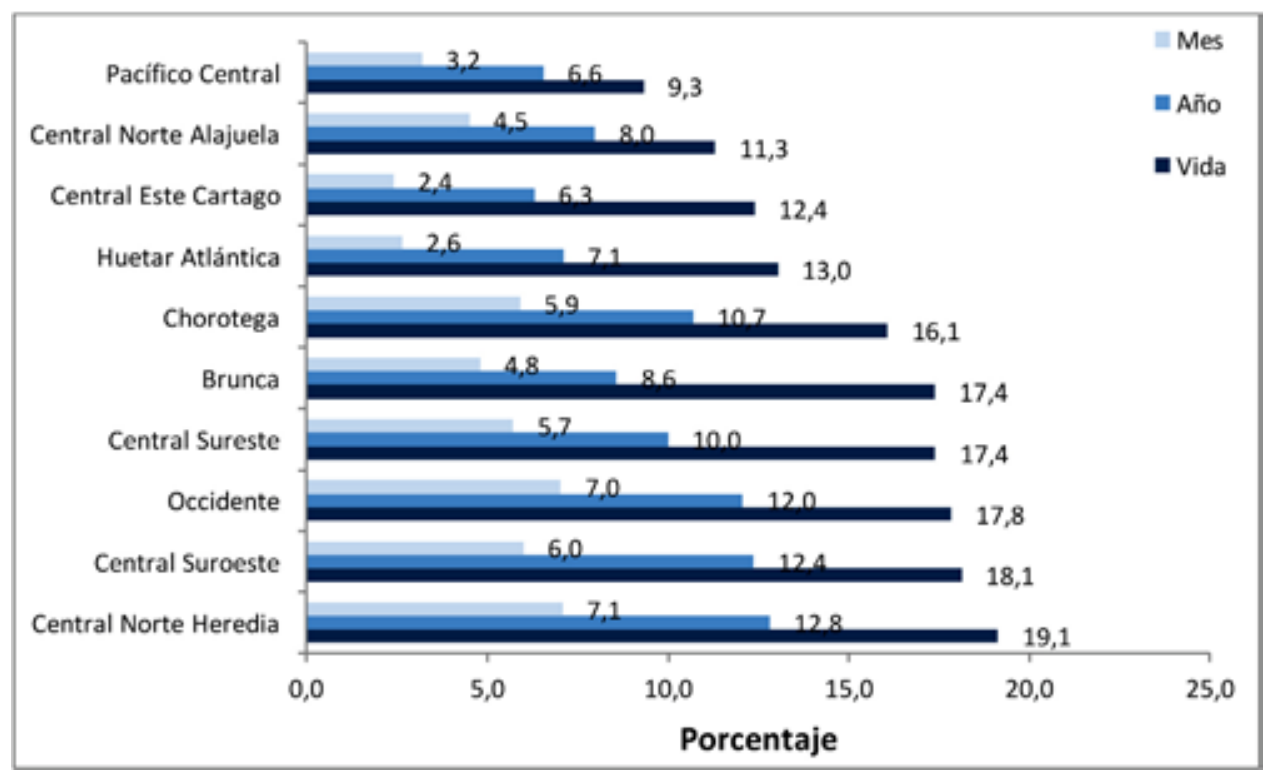

Fuente: IAFA, 2015.

El consumo de marihuana mostró particularidades interesantes tal y como se ha venido explicando en el desarrollo del documento, se puede apreciar que la región central norte, Heredia es la que presenta los tres indicadores más elevados, a pesar que, no son estadísticamente significativos en comparación con regiones como central sureste, occidente, central sureste, sí es evidente el mayor consumo en esta región de Heredia.

En este sentido, es importante mencionar que se ha mantenido una tendencia a la baja en la percepción de riesgo del consumo de esta droga, aquellos que consideran un mayor riesgo por consumir frecuentemente marihuana se ubican en $60,1 \%$, en comparación con los años 2009 (58,6\%) у $2012(66,6 \%)$. 
William Chacón Serrano. Geographical distribution of prevalence levels drug use in population of high school education in Costa Rica, 2015

\section{Conclusiones}

El análisis territorial del consumo de drogas en la población escolarizada sirve de punto de partida para establecer relaciones entre el consumo de drogas y las distintas regiones y provincias que se presentan en el país. Los resultados mostrados resultan atrayentes sobre el comportamiento de los niveles de prevalencia en ciertas zonas. En algunos casos se ven resultados que llaman la atención entre una región y otra, ya sea por factores geográficos, culturales, económicos, que se diferencian en los porcentajes de los indicadores; es decir, proporciones que se triplican entre una región y otra para el consumo de la misma droga.

La región Central Norte de Heredia es la que presenta indicadores más altos de consumo de alcohol, tabaco y marihuana. Las estimaciones reflejan claramente que las personas adolescentes escolarizadas que se ubican en esta región han experimentado un mayor uso de estas sustancias. En el estudio del 2012 el comportamiento fue parecido caracterizado por una tendencia a mayor uso en esta región lo mismo que para la provincia del mismo nombre (Heredia). La particularidad que se evidencia en estas zonas geográficas, tiene que ver con el índice de desarrollo humano cantonal, los cantones de esta provincia y región muestran mejores estimaciones de las condiciones de desarrollo humano. Una de las relaciones entre el consumo de drogas y los resultados en distintas regiones podría tener relación con el poder adquisitivo y lugares con alto desarrollo humano que dejan abierta la posibilidad de ahondar en estos campos en investigaciones puntuales en ciertas zonas específicas.

Por otra parte, las regiones Brunca y Atlántica presentan indicadores más bajos de consumo de drogas e igualmente siguiendo con la idea de párrafos anteriores, es importante averiguar las particularidades de estas regiones que las ubican sectores de consumo por debajo del promedio nacional; asimismo, otro aspecto que se puede mencionar son las disparidades regionales que se muestran para constatar el comportamiento del consumo, territorio y su distribución.

Los resultados obtenidos demuestran que el alcohol es la droga principal de consumo en la población escolarizada, seguido de la marihuana y el tabaco, resaltando el uso problemático en el caso del alcohol. Los hallazgos señalan que una cantidad importante de la población estudiantil consume alcohol de forma abusiva. El abuso de alcohol en la población 
adolescente representa un aspecto de recalcar, debido a que los estudiantes que consumen alcohol de forma abusiva están expuestos a una serie de problemas asociados que no solo afectan al consumidor, sino a las personas que los rodean. La problemática asociados al consumo abusivo de alcohol representa problemas de accidentabilidad de tránsito, violencia, abusos sexuales, en los cuales los jóvenes en muchos casos se ven involucrados

En cuanto a los aspectos negativos que asocian los estudiantes si se consumen drogas de forma frecuente, la percepción de riesgo representa un factor importante a la hora de analizar esta problemática del aumento en el consumo de las diferentes sustancias psicoactivas. Se pudo comprobar que el alcohol y la marihuana son drogas en las cuales la población estudiantil no manifiesta que exista un riesgo alto por consumir frecuentemente; es decir, los jóvenes no ven problemas físicos asociados al consumo de estas drogas, lo cual agrava las iniciativas que se presenten mediante políticas públicas por reducir el consumo. Estos resultados responden en parte al aumento en el consumo de marihuana y alcohol, al ser drogas socialmente permisivas la población ve ese comportamiento como parte del constructo social.

Por último, es importante resaltar que este tipo de ejercicios de monitoreo del consumo de drogas enfocados a las regiones del país, dan un aporte adicional a la investigación social, ya que el fenómeno se analiza directamente en un espacio geográfico determinado y permite la comparación de los resultados.

\section{Referencias}

Babor, T., Higgins-Biddle, J., Saunders, J. y Monteiro, M. (2001). AUDIT, El Test de Identificación de los Trastornos Debidos al Consumo de Alcohol. Generalitat Valenciana/ OMS. Recuperado de: http://www. who.int/substance_abuse/activities/en/AUDITmanualSpanish.pdf

Bejarano, J., Cortés, E., Chacón, W., Fonseca, S. López, K. y Sánchez, G. (2011).Juventud Escolarizada y drogas. Encuesta nacional sobre consumo de drogas en población de educación secundaria. San José: I.A.F.A. ISBN: 978-9968-705-84-4.

Comisión Interamericana para el Control del Abuso de Drogas (CICAD/ OEA). (2015). Informe sobre uso de drogas en las Américas 2015. Recuperado de internet el 21 de enero de 2015, recuperado de: http:// www.cicad.oas.org/apps/Document.aspx? $\mathrm{Id}=3209$ 
Fonseca, S. y Bejarano, J. (2012). Adolescentes costarricenses con problemas judiciales y consumo de drogas. Revista Costarricense de Psicología, 2012. 31, 1-2, p. 21-39. ISSN 0257-1439. Recuperado de: http://www.iafa.go.cr/investigacion/nuestras-investigaciones.html

Fonseca, S., Cortés, E., Chacón, W., Madrigal, S., Ortega, A., Salas, C. y Bejarano, J. (2012). Encuesta nacional sobre consumo de drogas en población de Educación secundaria: Costa Rica 2012. San José, Instituto sobre Alcoholismo y Farmacodependencia. ISBN: 978-9968705- $93-6$.

González, F., García, M. y González, S. (1996). Consumo de Drogas en la Adolescencia. Psicothema, 8(2), 257-267. Recuperado de: http:// www.redalyc.org/pdf/727/72780201.pdf

Instituto sobre Alcoholismo y Farmacodependencia. (2014). Análisis de Oferta Demanda en la atención a Personas Menores de Edad consumidoras problemáticas de sustancia psicoactivas en Costa Rica, 2012. Proceso de Investigación. San José, Costa Rica: IAFA, 2013. 978-9968-705-97-4

Instituto sobre Alcoholismo y Farmacodependencia. (2014). Análisis geográfico provincial del consumo de las drogas en Costa Rica 2011-2013. Proceso de Investigación. San José, Costa Rica: IAFA, 2013. Recuperado de: http://www.iafa.go.cr/investigacion/ nuestras-investigaciones.

Nazar, A., Tapia, R., Villa, A., León, G., Medina, M. y Salvatierra, B., (1994). Factores asociados al consumo de drogas en Adolescentes de áreas urbanas de México. Salud púbica de México, Recuperado de: http://bvs.insp.mx/rsp/_files/File/1994/num\%20\%20 6/199436_646-654.pdf.

Observatorio Argentino de Drogas (OAD) (2014). Sexto Estudio Nacional consumo de sustancias psicoactivas en Estudiantes de Enseñanza Media 2014. Recuperado de: http://www.observatorio.gob.ar/ www/547/19905/estudiantes-de-nivel-medio.

Oficina de las Naciones Unidas contra la Droga y el Delito. (UNODC) (2016). Abuso de drogas en adolescentes y jóvenes y vulnerabilidad familiar. Lima- Perú: Industrias Gráficas MACOLE S.R. Recuperado de: http://www.descentralizadrogas.gov.co/project/abuso-de-drogas-en-adolescentes-y-jovenes-y-vulnerabilidad-familiar/ 
Programa de Naciones Unidas para el Desarrollo/Universidad de Costa Rica. (PNUD/UCR). (2011). Atlas de desarrollo humano cantonal de Costa Rica, 2011. San José: Ediciones Sanabria, S.A.

Ramírez, M. (2009). La moderna geografía de la salud y las tecnologías de la información Geográfica. Revista investigaciones y ensayos geográficos, IV(4), 53-64, recuperado de: http://hum.unne.edu.ar/ investigacion/geografia/labtig/publicaciones/public17.pdf

Salamó, A., Grass, M. y Font, S. (2010). Patrones de consumo de alcohol en la adolescencia. Psicothema, 22(2), 189-195. Recuperado de: $\mathrm{http}: / / \mathrm{www} . r e d a l y c . o r g / p d f / 727 / 72712496003 . p d f$

UNICEF. (2011). Estado mundial de la infancia 2011. La adolescencia, una época de oportunidades. New York: Fondo de las Naciones Unidas para la Infancia (UNICEF) ISBN: 978-92-806-4557-6. 